\title{
Orbital magnetism of transition-metal adatoms and clusters on the $\mathrm{Ag}$ and $\mathrm{Au}(001)$ surfaces
}

\author{
I. Cabria, B. Nonas, R. Zeller, and P. H. Dederichs \\ Institut für Festkörperforschung, Forschungszentrum Jülich, D-52425 Jülich, Germany \\ (Received 16 February 2001; revised manuscript received 7 September 2001; published 9 January 2002)

\begin{abstract}
We present $a b$ initio calculations of the orbital moments and magnetocrystalline anisotropy energies for $3 d$, $4 d$, and $5 d$ transition-metal adatoms and for some selected small clusters on the (001) surfaces of Ag and Au. The calculations are based on the local density approximation of density functional theory and apply a fully relativistic Koringa-Kohn-Rostoker Green's function method. Due to the reduced coordination of the adatoms and the weak hybridization with the substrate, we find fairly large orbital moments, in particular for the elements towards the end of the series. The general trend can be understood from a simple tight-binding model. The orbital moments are connected with very large anisotropy energies. While the orbital moments are on the Ag substrate somewhat larger than on $\mathrm{Au}$, the magnetic anisotropy has about the same size for both substrates. Calculations for small clusters of $\mathrm{Fe}, \mathrm{Ru}$, and Os adatoms show, that due to interaction effects the orbital moments are strongly reduced, e.g., by $50 \%$ for the dimer atoms. The size of the reduction correlates well with the coordination number. Similar reductions also occur for the magnetic anisotropy energies.
\end{abstract}

DOI: 10.1103/PhysRevB.65.054414

PACS number(s): 75.70.Cn, 75.30.Gw, 71.70.Ej

\section{INTRODUCTION}

During the last decades, the developments of new experimental and theoretical techniques have enabled the study of clean surfaces and in particular the study of the magnetic properties of surfaces. In general, the spin moments were found to be enhanced at surfaces relative to their value in the bulk. This is the case, for instance, for the surface moments of the ferromagnets $\mathrm{Fe}, \mathrm{Co}$, and Ni. ${ }^{1,2}$ The usual explanation is that the coordination number is reduced at the surface, so that the hybridization with the neighboring atoms decreases. This causes a narrowing of the $d$ band, which enhances the spin moment. This enhancement of the spin moments has also been found in calculations of single adatoms, clusters, and monolayers of transition-metal (TM) atoms on noble surfaces. $^{3-9}$

For the same reason, one expects also an enhancement of the orbital moment $(\mathrm{OM})$ at surfaces. In addition to the mentioned reduction of the coordination number, the reduction of the symmetry at the surface can also partially unlock the quenching of the orbital moment induced by the crystal field. Some recent theoretical and experimental papers have reported this orbital moment enhancement. ${ }^{2,10-13}$ In absolute values, the OM enhancement is smaller than the spin moment enhancement, but the relative change of the orbital moments is much bigger. For instance, Hjortstom et al. found in their calculations that in bulk bcc Fe the spin and orbital moments are 2.25 and $0.049 \mu_{B}$, respectively, and 2.94 and 0.096 at the bcc Fe (001) surface. ${ }^{2}$ However, these enlarged orbital moments at surfaces are still much smaller than the corresponding free atom values, and hence, the orbital moment is basically still quenched at surfaces. Since closely related to the orbital moments, the magnetocrystalline anisotropy energies (MAE) are also of considerable interest in orbital magnetism, and similar reasons as for the orbital moment support the expectation of an enhancement of these energies at surfaces. By moving from the bulk to thin films, surfaces and interfaces, the MAE is usually enhanced by $2-3$ orders of magnitude. ${ }^{10,14-22}$
Due to recent progress in nanotechnology, researchers are now able to produce diverse novel nanostructures of TM adatoms on surfaces, opening on this way a new type of surface magnetism investigation. ${ }^{23}$ Clusters on surfaces are an intermediate step between monolayers and single atoms on surfaces. There are many experimental and theoretical studies of the magnetic properties of TM monolayers, clusters and single atoms on noble metal surfaces. ${ }^{7,9,24-31}$ In these former studies it was found that the magnetic moments of these structures on surfaces are enhanced compared to the moments of the bulk, and that their magnetic properties depend strongly on the type of substrate and, in the case of clusters, on the size and form of the clusters.

The purpose of this paper is to present $a b$ initio calculations of the magnetic properties of TM adatoms and small clusters on the Ag and Au surfaces, focusing on the orbital moments and MAE. We have performed fully relativistic calculations for all $3 d, 4 d$, and $5 d$ atoms as adatoms on the $\mathrm{Ag}$ and $\mathrm{Au}(001)$ surfaces and as substitutional impurities in fcc bulk $\mathrm{Ag}$ and in the first layer of the $\mathrm{Ag}$ surface. In addition we have considered some small clusters of the selected elements $\mathrm{Fe}, \mathrm{Ru}$, and $\mathrm{Os}$ on the $\mathrm{Ag}$ surface.

The next section gives a brief description of the theoretical method used in our study. In the third and fourth sections we will present the results of spin polarized relativistic (SPR) Korringa-Kohn-Rostoker (KKR) calculations of magnetic properties of TM single adatoms in and on the $\mathrm{Ag}$ and $\mathrm{Au}(001)$ surfaces as well as in bulk Ag. In Sec. V, ab initio results for small clusters of TM adatoms on the Ag surface are presented.

\section{DESCRIPTION OF THE THEORETICAL METHOD}

We have used the relativistic version of the KorringaKohn-Rostoker Green's function method for impurities on surfaces and in the bulk, based in the local spin density approximation (LSDA). The calculations of clusters on surfaces consist of three steps. The first one concerns the calculation of the bulk systems fcc $\mathrm{Ag}$ and $\mathrm{Au}$. In the second step, 


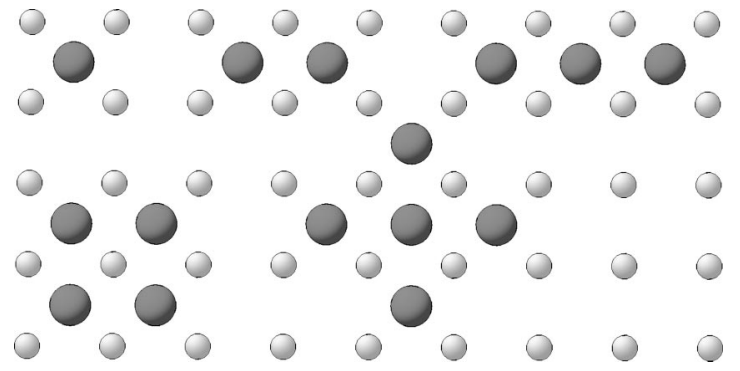

FIG. 1. Pictures of the clusters on the Ag fcc (001) surface studied in this work. The big dark spheres represent TM adatoms and the smaller white ones represent surface $\mathrm{Ag}$ atoms.

the ideal fcc (001) surface of Ag and Au are calculated, starting from the Green's function of the bulk as reference system. The surface is treated as a two-dimensional perturbation of the bulk and two half crystals are created by removing the potentials of eight monolayers. The Green's function of these half crystals, surfaces respectively, is obtained by solving an algebraic Dyson equation, using the Green's function of the bulk materials as reference and making full use of the twodimensional (2D) symmetry of this surface. In the third and final step, this new Green's function of the surface is used as reference system for the calculations of impurities on and in the surfaces. In the case of impurities in bulk, the bulk Green's function represents the input in the impurity calculation.

The impurities are put in two different positions: in the surface metal layer, replacing a silver or gold atom, and as adatoms at a "lattice site" in the first vacuum layer on the surface metal layer. In all the impurity calculations the perturbed system to be calculated self-consistently is a cluster formed by the TM atoms and the two or three nearest shells of neighbor sites (silver or gold substrate atoms or "empty sites" in the vacuum region, or silver bulk atoms). Hence, also the neighboring sites around the TM atoms are allowed to relax electronically. However, lattice relaxations are neglected in the calculations; the atoms remain fixed at the ideal lattice sites. Typically, a cluster of about 13-43 perturbed atomic potentials is determined self-consistently. The exchange-correlation potential of Vosko et $a .^{32}$ is used. The scattering potentials are assumed to be spherically symmetric, but the charge densities are expanded in multipoles up to $l=6$. For more details about the method see Refs. 7,26,27. Five different types of clusters on the surface are studied: one single adatom, a dimer, a linear trimer, a square and a cluster of five atoms (four atoms forming a cross and one atom in the middle of it). Figure 1 shows the geometrical structure of these clusters on the surface. Finally, the relativistic Lloyd's formula has been used to calculate total energy differences for different orientations of the magnetic moments of the whole cluster. ${ }^{33,34}$

\section{SPIN AND ORBITAL MOMENTS OF TRANSITION METAL ADATOMS}

In the following we present our results for the $3 d, 4 d$, and $5 d$ transition metal atoms on the (001) surfaces of $\mathrm{Ag}$

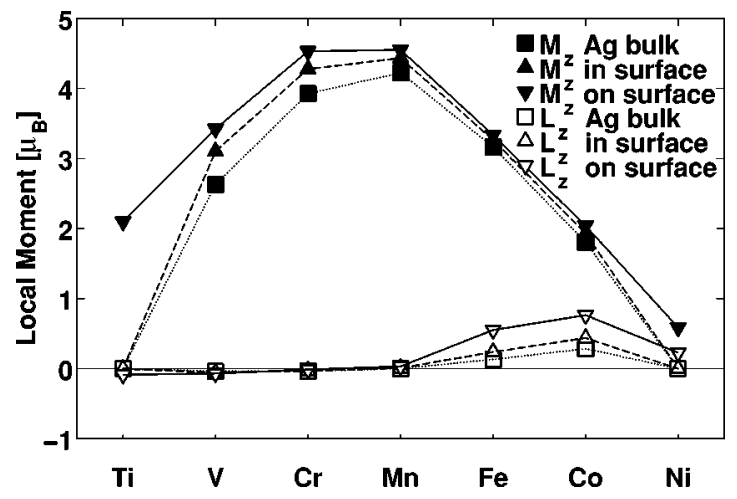

FIG. 2. Spin moments $M_{z}$ and orbital moments $L_{z}$ of the $3 d$ adatoms in bulk $\mathrm{Ag}$, in and on the $\mathrm{Ag}(001)$ surface, obtained in SPR calculations.

and $\mathrm{Au}$. The main emphasis will be on the enhancement of the orbital moment of the atoms at the surface. The data are the results of fully relativistic and self-consistent calculations using the LDA. In most calculations no orbital polarization term as in Ref. 35 has been used, so that the spin-orbit coupling is the only mechanism to create the orbital moments. The effect of Brooks' orbital polarization term ${ }^{36}$ on some of these results is discussed in Sec. VI. If not otherwise stated, the local moments are aligned perpendicular to the surface.

Figure 2 shows the local spin and orbital moments of single $3 d$ atoms for three different configurations: as adatoms on the $\operatorname{Ag}(001)$ surface, as substitutional impurities in the first surface layer and as impurities in bulk Ag. The major difference between these three configurations is the number of neighboring silver atoms. For the adatom, these are four, for a surface atom, eight, and for the bulk impurity, twelve. Accordingly, one finds that the adatoms have the largest spin moments and bulk atoms the smallest ones, with the values for the surface atoms in between. However, the differences are rather small, since already the spin moments of the bulk impurities are more or less "saturated." Significant differences only occur at the beginning and end of the series, since $\mathrm{Ti}$ and Ni have local moments as adatoms, but not as surface atoms or in the bulk.

The orbital moment shows a more complicated behavior, being for all configurations very small in the first half of the series, but significantly larger and strongly environmental dependent for the second half. In particular, the orbital moments of the $\mathrm{Fe}$ and Co adatoms are about a factor of three larger than the orbital moments of the bulk impurities.

It is instructive to decompose the orbital moments $L_{z}$ into the majority, $L_{z}$ up, and minority, $L_{z}$ down, contributions, being shown in Fig. 3. Since for the considered $3 d$ atoms, the exchange splitting is much larger than the width of the virtual bound states, we have a similar situation as for free atoms: First, the majority $d$ sublevels are filled up, starting with $m_{l}=-2,-1, \ldots$ This leads to negative moments, which vanish when the majority subshell is filled. Then the minority $d$ levels are populated in the reversed order $\left(m_{l}=+2,+1,0, \ldots\right)$. However, the difference with respect to the free atom is that the width of the virtual bound states of the adatoms is much larger than the spin-orbit splitting, at 


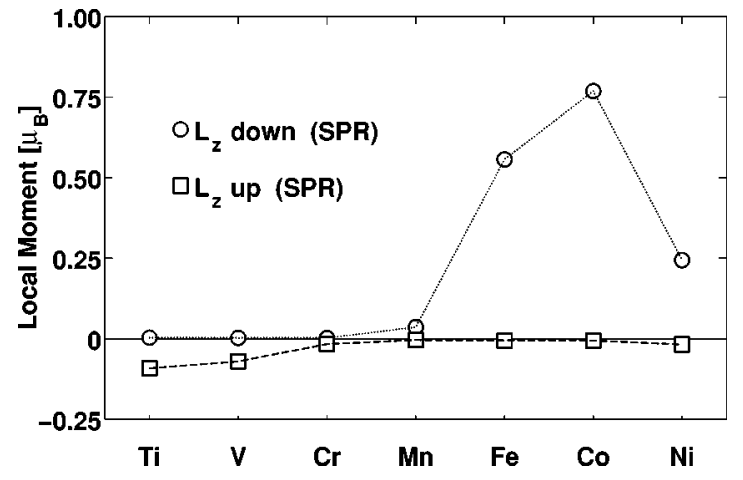

FIG. 3. Spin up and down orbital moments for $3 d$ adatoms on the $\operatorname{Ag}(001)$ surface, obtained in SPR calculations.

least for the early $3 d$ elements. Therefore, only very small negative orbital moments are obtained for $\mathrm{Ti}, \mathrm{V}$, and $\mathrm{Cr}$. At the end of the series, the virtual bound state have considerably narrowed, while the spin-orbit coupling (SOC) increases slightly. As a result, rather big and positive orbital moments are obtained for $\mathrm{Fe}, \mathrm{Co}$, and $\mathrm{Ni}$ adatoms, arising solely from the minority states.

Qualitatively the trends for the calculated orbital moments as presented in Fig. 3 can be understood, if one treats the spin-orbit term in first order perturbation theory. In this order, the spin-orbit perturbation $\Delta V$, given by

$$
\Delta V=\frac{\xi}{2} \vec{L} \cdot \vec{\sigma} \simeq \frac{\xi}{2} L_{z} \sigma_{z}
$$

can be replaced by $\Delta V \simeq \xi L_{z} \sigma_{z} / 2$, so that the spin is still a good quantum number. In a simple tight binding model proposed by Ebert et al. ${ }^{37}$ one obtains

$$
L_{z} \simeq-\xi\left[\stackrel{\circ}{n}\left(E_{F}\right)-\stackrel{\circ}{n} \downarrow\left(E_{F}\right)\right],
$$

where $\stackrel{\circ}{n}^{\uparrow}$ and $\stackrel{\circ}{n}^{\downarrow}$ are the spin up and down local density of states at the Fermi energy $E_{F}$ for the considered adatoms. This naturally explains all the above findings. For instance, due to the reduced coordination, the adatoms have narrower virtual bond states and higher densities of states, and thus larger orbital moments. Analogously, at the end of the series the widths of the virtual bound states are narrower, so that the orbital moments become larger, while the sign changes from negative to positive, since then the minority states are filled.

The validity of this model is illustrated in Fig. 4 for the orbital moments of $5 d$ adatoms on $\operatorname{Ag}(001)$ surface. Shown are the orbital moments as calculated from Eq. (2) with the spin-orbit parameter taken from Ref. 38 and the local density of states obtained from a scalar relativistic treatment, as compared to the results of a perturbative $a b$ initio calculation, i.e., one iteration of the Dirac-Kohn-Sham equations with converged scalar relativistic potentials as input. Thus, the trends are very well described by the above model. A selfconsistent fully relativistic calculation gives practically the same results, except for Ir, the spin and orbital moments of which vanish in a self-consistent treatment.

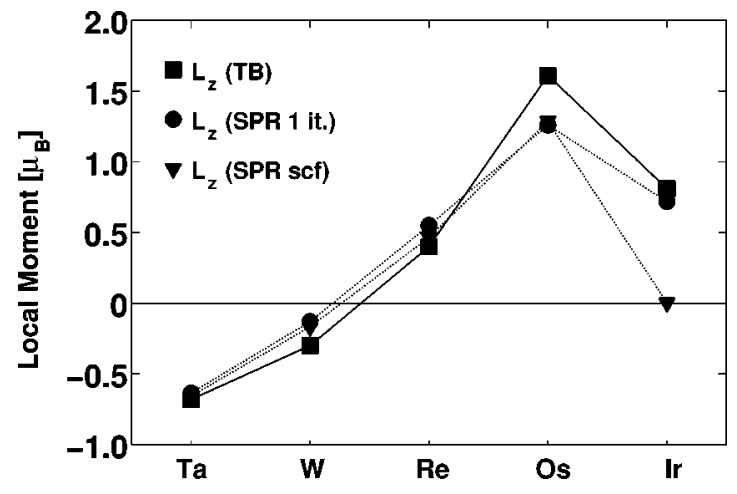

FIG. 4. Orbital moments of single $5 d$ adatoms on the $\operatorname{Ag}(001)$ surface calculated using the tight-binding (TB) model and in SPR non-self-consistent (1 iteration) and self-consistent calculations.

Equation (2) is also interesting when one compares the orbital moments for the three TM series. The increase of the spin-orbit parameter $\xi$ from the $3 d$ to the $4 d$ and then to the $5 d$ series is to a large extent counterbalanced by a decrease of the densities of states, induced by the increased hybridization of the $4 d$ and even more of the $5 d$ wave functions with the Ag $s p$ states. Therefore one finds that the largest orbital moments in the $4 d$ series [see below, Fig. 5(b), for Ru] have the same order of magnitude as the one of Fe and Co [Fig. $5(\mathrm{a})]$, while in the $5 d$ series the increased spin-orbit coupling wins, leading for the Os adatom to the largest orbital moment of all TM atoms. In contrast to the orbital moments, the spin moments are, due to the strong hybridization, reduced in the $4 d$ series and even more in the $5 d$ one [see Fig. 5(c)].

To discuss the influence of the substrate, we compare in Figs. 5(a), 5(b), and 5(c) the local spin and orbital moments of TM adatoms on the Au and Ag (001) surfaces. From electronic structure point of view, gold and silver are both noble metals and quite similar, with the major difference arising from the $d$ band, which is considerably closer to the Fermi level in gold and due to the stronger $d$ - $d$ hybridization and the larger spin-orbit splitting, also considerably broader. As we can see from Fig. 5, the differences in the moments on $\mathrm{Ag}$ and $\mathrm{Au}(001)$ are small for the $3 d$ atoms in Fig. 5(a), but become larger for the $4 d$ adatoms [Fig. 5(b)] and are largest for the $5 d$ ones [Fig. 5(b)]. This results from the larger extent of the $4 d$ and $5 d$ wave functions, which lead to a larger environmental sensitivity. As compared to $\mathrm{Ag}$, in all cases the spin and orbital moments are reduced on the Au surface due to the stronger hybridization with the $d$ band of the substrate. Moreover, one observes for the Au substrate a shift of the maximum in the spin moments to larger valences which can be explained by the covalent bonding of the impurity $d$ with the Au $d$ states, resulting to a small shift of the virtual bound states to higher energies. Similar results have also been obtained for substitutional TM impurities in the first layer of $\mathrm{Ag}$ and $\mathrm{Au}(001)$ surfaces. Compared to the adatoms, these spin and orbital moments are reduced, with the reduction being larger for the Au substrate.

Unfortunately, our present computational method does not allow one to include lattice relaxations. Without doubt such relaxations will be important for adatoms, in particular for the relatively small $3 d$ atoms on the $\operatorname{Ag}(001)$ surface. To get 

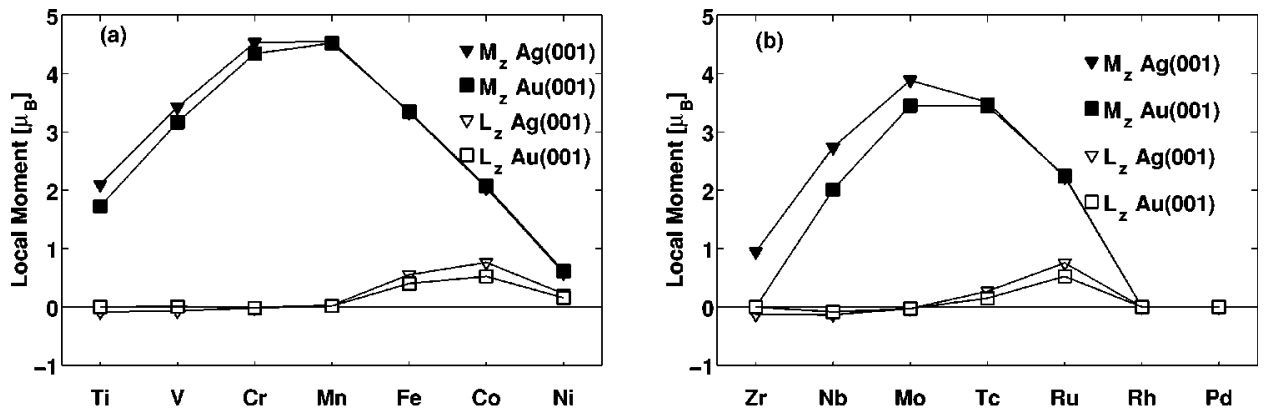

FIG. 5. Spin and orbital moments of the TM adatoms on the $\mathrm{Ag}$ and $\mathrm{Au}$ (001) surfaces, obtained in SPR calculations. (a) $3 d$

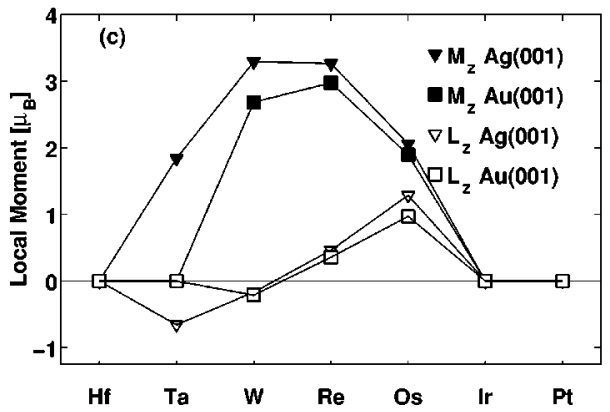
adatoms, (b) $4 d$ adatoms, and (c) $5 d$ adatoms.

an idea of the changes to be expected by lattice relaxations we consider the dependence of the orbital moments of the $3 d$ atoms on the coordination number (Fig. 2). Naturally we expect that even a relaxed $3 d$ adatom (with four Ag neighbors) should have a larger orbital moment than the (unrelaxed) $3 d$ atom in the surface layer (with eight Ag neighbors), and analogously that the relaxed surface atom should have a larger orbital moment than the (unrelaxed) $3 d$ impurity in the bulk (with $12 \mathrm{Ag}$ neighbors). Thus the calculated orbital moment for the $3 d$ surface atom should be a lower bound for the value of the relaxed $3 d$ adatom, and analogously the calculated value for the bulk impurity should be a lower bound for the orbital moment of the surface atom.

\section{MAGNETIC ANISOTROPY ENERGIES OF ADATOMS}

Due to the spin-orbit interaction the magnitude of the spin and orbital moments, as well as of the total energies, depends on the relative orientation of the moments with respect to the crystal axis. By treating the spin-orbit coupling as a perturbation, the resulting anisotropy energy of surfaces depends quadratically on the components $\alpha_{i}$ of the magnetization direction, $\vec{M}=\left(\alpha_{x}, \alpha_{y}, \alpha_{z}\right)$ relative to the crystal axes $\vec{x}, \vec{y}$ and $\vec{z}$ and scales quadratically with the spin-orbit coupling parameter. Two different direction dependences can occur: For a surface with a fourfold or threefold axis, such as the (001) or (111) surface of a cubic crystal, the anisotropy energy

$$
\Delta E=K_{1}^{s} \sin ^{2} \theta
$$

is quadratic in $\sin \theta$, with $\theta$ being the angle between the surface normal and the magnetization direction $\vec{M}$. This expression is also valid for adatoms or small clusters on these surfaces, as long as the point symmetry of these structures includes a threefold or fourfold axis, as it is, e.g., the case for a single adatom in the hollow site on the (001) surface, to be examined in this section.
For surfaces with lower symmetry, as, e.g., the (110) surface of cubic crystals, the anisotropy energy has an additional dependence on the azimuth $\phi$ in the surface plane

$$
\Delta E=K_{1}^{s} \sin ^{2} \theta+K_{2}^{s} \sin ^{2} \theta \cos 2 \phi,
$$

leading to an "uniaxial in-plane anisotropy." The same dependence is also obtained for clusters on the (001) or (111) surfaces, if the clusters destroy the $C_{3}$ or $C_{4}$ symmetry axes of these surfaces. This is the case for the adatom dimers or trimers to be discussed in the next section.

In test calculations for single $\mathrm{Co}$ and $\mathrm{Re}$ adatoms in the hollow site on the $\mathrm{Ag}(001)$ surface, as well as for substitutional $\mathrm{Co}$ and $\mathrm{Re}$ atoms in the first surface layer, we have examined the predicted $\sin ^{2} \theta$ dependence. We found that the MAE as well as the anisotropic contributions of the spin and orbital moments obey well the $\sin ^{2} \theta$ law and exhibit no appreciable $\phi$ dependence in the surface plane. The small deviations from these dependences found in the calculations, indicate that the residual fourth order anisotropy constants are two orders of magnitude smaller and can be safely neglected. Therefore, we discuss in the following only the perpendicular and in-plane configurations of the adatom moments.

In Fig. 6(a) we present the calculated anisotropy of the orbital moments (AOM) for TM adatoms on the $\mathrm{Au}(001)$ surface. Figure 6(b) represents the anisotropy of the spin moments for the same systems. Negative (positive) values mean, that the perpendicular moments are larger (smaller) than the parallel ones. First, the changes of the spin moments are very small, typically an order of magnitude smaller than the changes of the orbital moments. Secondly, the orbital curves for all three series follow the same trend, but with a size signalizing the increase of the SOC parameter $\xi$ from the $3 d$ to the $5 d$ series. Thirdly, positive values are obtained at the beginning and the end of the series for the anisotropy of the orbital moment, while in the middle negative values 

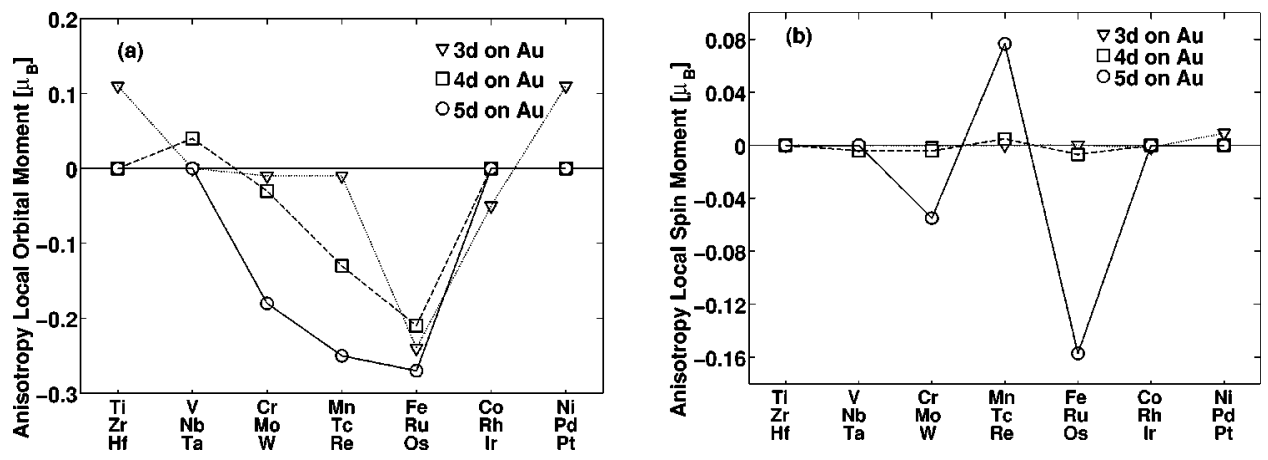

FIG. 6. Anisotropy of the orbital moment (a) and spin moment (b) of TM adatoms on the $\mathrm{Au}(001)$ surface, obtained in SPR calculations. prevail. Similar trends are also obtained for the silver surface, with only slight deviations.

Figures 7(a), 7(b), and 7(c) show the calculated MAE $\left(K_{1}^{s}\right)$ for $3 d, 4 d$, and $5 d$ adatoms, respectively, on the $\mathrm{Ag}$ and $\mathrm{Au}$ surfaces, with positive (negative) values meaning perpendicular (in-plane) orientations preferred. The calculated energies are of the order of 1-30 $\mathrm{meV}$, with particularly the $5 d$ values being an order of magnitude larger than the anisotropies per TM atom of TM monolayers and multilayers. The large $5 d$ values are clearly related to the large SOC parameter of these elements. For both substrates the size of the anisotropy is quite similar, despite the fact that the orbital moments are always smaller for the gold substrate. From Figs. 7(a), 7(b), and 7(c) one observes that the MAE shows an oscillatory behavior in each series, which has been explained by Pick and Dreyssé. 6

In general we found that the MAE shows, for the systems investigated, no clear relation to the orbital moments nor to the anisotropy of the orbital moments as shown in Fig. 7(a). A direct relation to the anisotropy Bruno's formula ${ }^{39}$ or the improved version of van der $\operatorname{Laan}^{40}$ is also not found. The reason is that, in particular for the $4 d$ and $5 d$ adatoms, a perturbative determination of the MAE by the force theorem is not valid in general. This would mean a determination of the MAE from the single particle energies using the spin- polarized scalar relativistic potentials as input. While this gives good results for the $3 d$ atoms, for the $4 d$ and $5 d$ atoms the increased SOC and the stronger hybridization with the substrate require a self-consistent relativistic treatment by including the neighboring Ag or Au atoms. Here, also the magnetic effects are not sufficiently localized at the impurity and a significant contribution to the MAE arises from the neighboring silver or gold atoms. For an accurate evaluation of these terms the inclusion of a large cluster of perturbed potentials as well as the use of Lloyd's formula for the single particle energies, as we have done, is essential.

\section{TRANSITION METAL CLUSTERS ON THE Ag(001) SURFACE}

Due to interaction effects the local moments of TM atoms in a cluster are different from the ones of single adatoms. Hence, in addition to the substrate, also the size and the geometrical structure of the cluster influences the atomic moments. Due to the large manifold of possible clusters, we restrict ourselves to small clusters with up to 5 adatoms. Moreover, we consider only clusters of the isoelectronic elements Fe ( $3 d$ series), Ru (4d), and Os $(5 d)$. These elements have been chosen, since the corresponding adatoms have one of the largest MAE and orbital moments in the

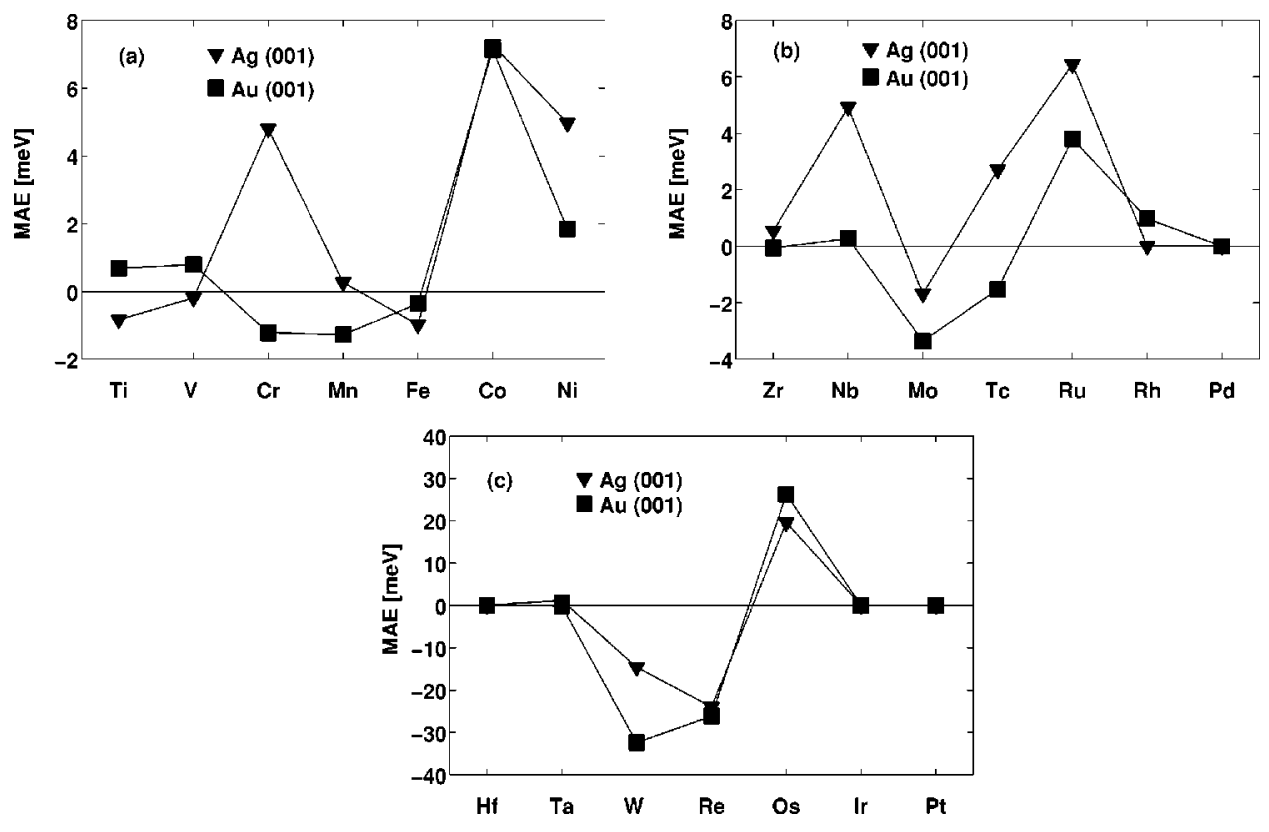

FIG. 7. Magnetic anisotropy energy $K_{1}^{s}$ for TM adatoms on the $\mathrm{Ag}(001)$ and $\mathrm{Au}(001)$ surfaces, obtained in SPR calculations. (a) $3 d$ adatoms, (b) $4 d$ adatoms, and (c) $5 d$ adatoms. Positive values mean perpendicular orientation. 
TABLE I. Spin moment, orbital moment, and anisotropy of the orbital moment $\Delta L$ (in $\mu_{B} /$ atom) of TM adatoms (in $\mu_{B}$ /atom) and magnetocrystalline anisotropy energy per TM atom $\Delta E$ (in $\mathrm{meV}$ ) of transition metal clusters on the $\mathrm{Ag}(001)$ surface obtained in SPR-KKR calculations.

\begin{tabular}{|c|c|c|c|c|c|c|c|c|c|}
\hline Symbol & $\begin{array}{l}\text { Number of } \\
\text { TM adatoms }\end{array}$ & $N_{c}$ & $\begin{array}{l}\text { Type of } \\
\text { atom }\end{array}$ & $M_{z}$ & $L_{z}$ & $\Delta L_{x-z}$ & $\Delta E_{x-z}$ & $\Delta L_{y-z}$ & $\Delta E_{y-z}$ \\
\hline \multirow[t]{9}{*}{$\mathrm{Fe}$} & 1 & 0 & & 3.33 & 0.55 & -0.20 & -0.98 & & \\
\hline & 2 & 1 & & 3.28 & 0.28 & -0.06 & +0.28 & -0.07 & -0.42 \\
\hline & 3 & 2 & Central & 3.26 & 0.22 & -0.03 & & -0.05 & \\
\hline & & 1 & External & 3.26 & 0.35 & -0.08 & & -0.09 & \\
\hline & & 1.3 & Average & 3.26 & 0.30 & -0.06 & +0.30 & -0.08 & +0.04 \\
\hline & 4 & 2 & & 3.21 & 0.20 & -0.03 & +0.12 & & \\
\hline & 5 & 4 & Central & 3.10 & 0.14 & -0.00 & & & \\
\hline & & 1 & External & 3.29 & 0.32 & -0.05 & & & \\
\hline & & 1.6 & Average & 3.25 & 0.28 & -0.04 & -0.09 & & \\
\hline \multirow[t]{9}{*}{$\mathrm{Ru}$} & 1 & 0 & & 2.23 & 0.75 & -0.19 & +6.44 & & \\
\hline & 2 & 1 & & 2.19 & 0.36 & -0.07 & +2.54 & +0.09 & +3.37 \\
\hline & 3 & 2 & Central & 2.17 & 0.27 & -0.05 & & +0.25 & \\
\hline & & 1 & External & 1.97 & 0.42 & -0.05 & & +0.06 & \\
\hline & & 1.3 & Average & 2.04 & 0.37 & -0.05 & +1.05 & +0.12 & +2.35 \\
\hline & 4 & 2 & & 1.85 & 0.17 & +0.07 & -0.48 & & \\
\hline & 5 & 4 & Central & 1.68 & 0.12 & +0.06 & & & \\
\hline & & 1 & External & 2.05 & 0.43 & +0.02 & & & \\
\hline & & 1.6 & Average & 1.98 & 0.37 & +0.03 & +1.95 & & \\
\hline \multirow[t]{9}{*}{ Os } & 1 & 0 & & 2.06 & 1.28 & -0.25 & +21.28 & & \\
\hline & 2 & 1 & & 1.38 & 0.56 & -0.16 & +23.06 & +0.03 & +71.20 \\
\hline & 3 & 2 & Central & 0.00 & 0.00 & +0.00 & & +0.00 & \\
\hline & & 1 & External & 0.00 & 0.00 & +0.00 & & +0.00 & \\
\hline & & 1.3 & Average & 0.00 & 0.00 & +0.00 & +0.00 & +0.00 & +0.00 \\
\hline & 4 & 2 & & 0.00 & 0.00 & +0.00 & +0.00 & +0.00 & +0.00 \\
\hline & 5 & 4 & Central & -0.05 & 0.05 & -0.12 & & & \\
\hline & & 1 & External & 1.14 & 0.54 & +0.09 & & & \\
\hline & & 1.6 & Average & 0.90 & 0.44 & +0.05 & +3.44 & & \\
\hline
\end{tabular}

corresponding series. While the MAE of the single adatoms consists of the magnetocrystalline contribution, for the clusters in addition a dipolar contribution exists, which is, however, vanishingly small. In Fig. 1 the considered clusters, which are more or less compact, are schematically shown. The results of the calculations are summarized in Table I. For dimers and trimers there are two different anisotropies depending on the orientation of the moments in the plane with respect to the main axis of these clusters. The axes of these clusters are oriented in the $y$ axis. In Table I the subindex $x-z(y-z)$ means that the quantity considered is the difference between two values, the first one related to a configuration with all moments in the plane and along the $x(y)$ axis and the second one $z$ related to the configuration with all moments perpendicular to the surface plane.

Dimers. Compared to the single adatoms, the atomic moments in the $\mathrm{Fe}$ and $\mathrm{Ru}$ dimers are only slightly reduced. Apparently the dimerization induced splitting of the virtual bound states is still smaller than the exchange splitting, so that due to charge neutrality, the moment will not change. In contrast to this, the atomic orbital moments are in both cases reduced by a factor of 2 . For $\mathrm{Os}_{2}$ we see, on the other hand, an appreciable reduction of the spin moment (by 33\%), but also here the reduction of the orbital moment is even larger $(56 \%)$ than for $\mathrm{Fe}_{2}$ and $\mathrm{Ru}_{2}$. Note that not only for the dimers, but also for all the clusters of $\mathrm{Fe}$ and $\mathrm{Ru}$, the present fully relativistic calculations yield practically the same moments as previous scalar relativistic KKR results. ${ }^{7,26,27}$ However, the Os moment is considerably reduced, i.e., partly quenched by the larger spin-orbit splitting. Similar to the Fe adatom, the $\mathrm{Fe}_{2}$ dimers have in-plane moments oriented along the dimer axis. In contrast to this, for the $\mathrm{Ru}$ and $\mathrm{Os}$ dimers the perpendicular orientation is the most stable one. Moreover the atomic anisotropy of the Os dimer is even larger than the anisotropy of the single Os adatom.

Trimers. For the linear trimers (Fig. 1) we find that the local spin moments of $\mathrm{Fe}_{3}$ are practically unchanged, while the spin moments of $\mathrm{Ru}_{3}$ are somewhat reduced, compared to the dimers. A drastic effect occurs for the $\mathrm{Os}_{3}$ trimer which turns out to be nonmagnetic, while previous scalar relativistic calculations yielded an average moment of $1.43 \mu_{B}$. Thus the broadening of the density of states due to spin-orbit coupling has killed the moment. Typical for the orbital moment of $\mathrm{Fe}_{3}$ and $\mathrm{Ru}_{3}$ is that the twofold coordi- 
nated central atoms have smaller orbital moments than the single coordinated outer atoms. According to the calculated MAE the $\mathrm{Fe}_{3}$ and $\mathrm{Ru}_{3}$ trimers prefer a perpendicular orientation. However, for the $\mathrm{Fe}_{3}$ trimer the perpendicular orientation is nearly degenerate with the in-plane orientation along the trimer axis. For both, the $\mathrm{Fe}_{3}$ and the $\mathrm{Ru}_{3}$ trimer, the anisotropy energy per TM atom and also the total anisotropy energy are strongly reduced.

Tetramers. The considered tetramer has the form of a compact square, where each atom has the coordination 2. Compared to the trimers, the spin moments are further reduced, less for $\mathrm{Fe}$ and more for Ru. Similar to the trimer, the Os tetramer is nonmagnetic, while a SRA-KKR calculation yielded local moments of about $1.22 \mu_{B}$. For Fe and $\mathrm{Ru}$, the reduction of the orbital moments and MAE from the trimer values is again much larger than the reduction of the spin moments, showing the extreme sensitivity of these quantities with respect to the hybridization strength. The moments are in-plane for $\mathrm{Ru}_{4}$ and out-of-plane for $\mathrm{Fe}_{4}$.

Pentamers. The considered pentamer (Fig. 1) is basically an open structure, since the four outer atoms have only one nearest neighbor, while the central atom is fourfold coordinated. We find, therefore, for $\mathrm{Fe}$ as well as $\mathrm{Ru}$, a smaller spin moment for the central atom and somewhat larger moments for the outer ones. This behavior is very extreme for the Os pentamer, showing a spin moment of $1.14 \mu_{B}$ for the external atoms, but a nearly vanishing spin moment for the central one. Again this trend is exaggerated for the orbital moments, which are in all three cases very small for the central atoms. The outer atoms have the same coordination as the dimer atoms. Indeed, we find a strong similarity between the spin as well as the orbital moments of these outer atoms and the dimer atoms. Thus, the large spin and orbital moments of the outer atoms are a direct consequence of the open structure of the considered pentamer and this shows also up in the relatively high anisotropy energies. The Os and Ru pentamer moments point out-of-plane, but the Fe pentamer is in-plane.

Concluding this section, we note that the orbital moments and anisotropy energies per atom are, in general, strongly reduced upon clustering. Already for the dimers the orbital moments are less than half the orbital moments of the single adatoms and the anisotropies are even smaller. Moreover, we find a strong sensitivity of the orbital moments and of the anisotropy of the orbital moments on the local coordination number, so that high coordinated sites have very small orbital moments and low coordinated sites have larger orbital moments. The same is also true for the anisotropy energy, if low coordinated sites prevail.

\section{EFFECTS OF ORBITAL POLARIZATION}

The spin-orbit coupling, being included in the Dirac equation, is not the only physical effect, which induces an orbital moment. As is known from the free atoms, there exists also an intraatomic orbital polarization (OP) effect of electrostatic origin being responsible for the maximal orbital moments as given by Hund's second rule. Since this effect cannot be described by the local-density approximation (LDA) densityfunctional theory, Brooks ${ }^{36}$ has suggested a way to improve

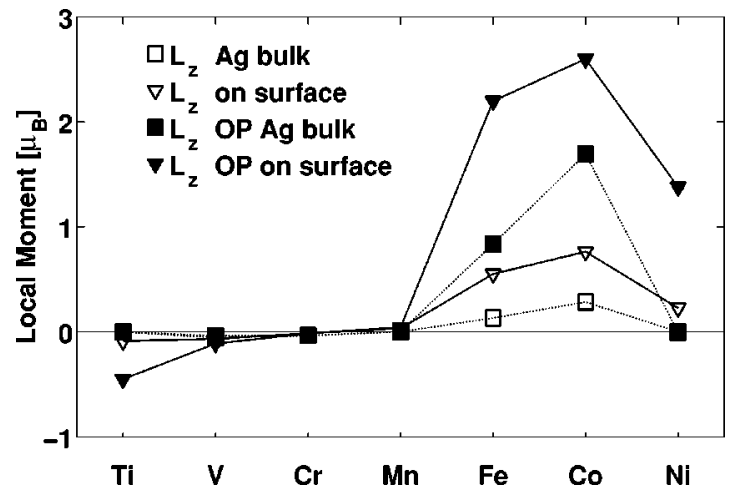

FIG. 8. Orbital moments of $3 d$ adatoms on the $\mathrm{Ag}(001)$ surface and of $3 d$ impurities in bulk Ag. Closed symbols are for results which include the orbital polarization OP term and open symbols are for LDA results without the OP term.

this shortcoming by adding an additional heuristic term in the Hamiltonian being proportional to the Racah parameter times the square of the orbital moment. This term enhances the calculated orbital moments and anisotropy energies, both in the bulk and at surfaces, ${ }^{13}$ and leads to better agreement with experiments. In a recent paper ${ }^{41}$ we have presented calculations, using the OP formula of Ebert et al. ${ }^{35}$ for the orbital moments and anisotropies of $3 d$ and $5 d$ adatoms on the $\mathrm{Ag}(001)$ surface. For the $3 d$ atoms $\mathrm{Fe}, \mathrm{Co}$, and $\mathrm{Ni}$ we find extremely large orbital moments, e.g., $2.57 \mu_{B}$ for the Fe adatom, which are much larger than the LDA values (e.g., $0.76 \mu_{B}$ for the $\mathrm{Fe}$ adatom). These large values even survive, if we switch off spin-orbit splitting, i.e., in a scalar relativistic treatment but including the OP term, which we have interpreted as a localized behavior of the Fe adatoms. For the early $3 d$ elements, as well as for the $4 d$ and $5 d$ adatoms the OP enhancement is more normal, i.e. similar as in the bulk ferromagnets.

Here we supplement the earlier results for the $3 d$ adatoms on $\operatorname{Ag}(001)$ (Ref. 41) by analogous results for the $3 d$ impurities in Ag. Figure 8 shows the orbital moments for the $3 d$ adatoms and the $3 d$ impurities calculated in the fully relativistic scheme with and without the orbital polarization term. (The spin moments calculated both ways are practically identical.) To our surprise we find that also the impurity moments are strongly enhanced by the OP term, in particular the values of $\mathrm{Fe}\left(0.8 \mu_{B}\right)$ and Co impurities $\left(1.7 \mu_{B}\right)$. The reason for this unusual behavior can be traced to the narrow minority virtual bound states of the $\mathrm{Fe}$ and Co impurities. In the case of Co this peak is exactly at the Fermi level and has a halfwidth which is considerably smaller than the OP induced splitting. ${ }^{41}$ Therefore a very large orbital moment results, which even survives, if the spin-orbit coupling is switched off so that a "spontaneous" orbital moment occurs induced only by the OP term.

Thus the OP scheme yields for the Fe and Co impurities very large orbital moments, which are not much smaller than the one obtained for the corresponding adatoms. Unfortunately, we do not know any susceptibility or hyperfine field measurements for Co impurities in Ag. However for Fe impurities in Ag Mössbauer data by Steiner and Hüfner ${ }^{42}$ exist, 
yielding a hyperfine field of $-33 \mathrm{kG}$ and a local moment of $3.1 \mu_{B}$. While this local moment agrees well with the calculated LDA moment of $3.29 \mu_{B}$ consisting of a spin moment of $3.16 \mu_{B}$ and an orbital moment of $0.13 \mu_{B}$, it disagrees with the OP result of $4.0 \mu_{B}$, since the orbital moment increases strongly $\left(0.83 \mu_{B}\right)$. Also the experimental hyperfine field of $-33 \mathrm{kG}$ agrees reasonably well with the LDA value of $-71 \mathrm{kG}$ for the Fe impurity, whereas the field calculated within the OP scheme becomes positive $(349.7 \mathrm{kG})$ due to the large positive orbital hyperfine contribution of $486 \mathrm{kG}$ arising from the strongly enhanced orbital moment. We conclude that the orbital moments of the $3 d$ impurities and maybe also of the $3 d$ adatoms are strongly overestimated by the OP term, presumably due to the large density of states at $E_{F}$, leading to a spontaneous orbital polarization. We also calculated the effect of Brooks' OP term for small Fe clusters on $\mathrm{Ag}$ and found enhancements factors between 5 and 6 for the orbital polarization of all $\mathrm{Fe}$ atoms in dimer, trimer, tetramer, and pentamer configurations, which seem to be unreasonably high. Thus for the present impurity systems, being characterized by a very high density of states at $E_{F}$, the OP term of Brooks gives unreliable results.

\section{CONCLUSIONS}

Our calculations based on the local density approximation and a fully relativistic Green's function method yield a large enhancement of the orbital moments for single TM atoms as adatoms on the (001) surfaces of $\mathrm{Ag}$ and $\mathrm{Au}$. For all three series, the orbital moments are largest toward the end of the series. The general trends for the orbital moments can be explained by a simple tight-binding model, yielding a proportionality to the spin-orbit coupling parameter and the local spin polarization at the Fermi energy. While the anisotropy of the orbital moments shows for all three series a well defined trend, the MAE is more irregular and shows no direct correlation with the anisotropic part of the orbital moments. On both substrates, a similar behavior is found, with the spin and orbital moments being somewhat smaller on the Au substrate. For adatoms of all three series, very large magnetic anisotropy energies are obtained, with the largest one occurring for the $5 d$ elements. Contrary to the moments, the anisotropy energies have the same magnitude on both substrates. The calculations for small clusters of $\mathrm{Fe}, \mathrm{Ru}$, and $\mathrm{Os}$ adatoms show that due to interaction effects between the adatoms, the orbital moments are strongly reduced, e.g., by a factor of 2 for the dimer atoms. The size of the reduction correlates well with the coordination number. Similar reductions also occur for the magnetic anisotropy energy, although this behavior is more irregular.

\section{ACKNOWLEDGMENTS}

We thank Stefan Blügel for helpful discussions concerning the magnetic anisotropy energy. In particular we thank Hubert Ebert and Till Huhne for their help and advice in producing the fully relativistic KKR-Green's function program. This work was partially funded by the Schwerpunktprogramm "Relativistische Effekte" of the Deutsche Forschungsgemeinschaft and the TMR network "Interface Magnetism" of the European Union.
${ }^{1}$ S. Ohnishi, A. J. Freeman, and M. Weinert, Phys. Rev. B 28, 6741 (1983).

${ }^{2}$ O. Hjortstam, J. Trygg, J. M. Wills, B. Johansson, and O. Eriksson, Phys. Rev. B 53, 9204 (1996).

${ }^{3}$ S. Blügel, B. Drittler, R. Zeller, and P. H. Dederichs, Appl. Phys. A: Solids Surf. A49, 547 (1989).

${ }^{4}$ S. Blügel and P. H. Dederichs, Europhys. Lett. 9, 597 (1989).

${ }^{5}$ O. Eriksson, R. C. Albers, and A. M. Boring, Phys. Rev. Lett. 66, 1350 (1991).

${ }^{6}$ S. Pick and H. Dreyssé, Phys. Rev. B 46, 5802 (1992).

${ }^{7}$ P. Lang, V.S. Stepanyuk, K. Wildberger, R. Zeller, and P. H. Dederichs, Solid State Commun. 92, 755 (1994).

${ }^{8}$ B. Újfalussy, L. Szunyogh, and P. Weinberger, Phys. Rev. B 51, 12836 (1995).

${ }^{9}$ V. S. Stepanyuk, W. Hergert, K. Wildberger, R. Zeller, and P. H. Dederichs, Phys. Rev. B 53, 2121 (1996).

${ }^{10}$ G. H. O. Daalderop, P. J. Kelly, and M. F. H. Schuurmans, Phys. Rev. B 44, 12054 (1991).

${ }^{11}$ T. Huhne, C. Zecha, H. Ebert, P. H. Dederichs, and R. Zeller, Phys. Rev. B 58, 10236 (1998).

${ }^{12}$ S. Y. Park, S. Muto, A. Kimura, S. Imada, Y. Kagoshima, T. Miyahara, T. Hatano, T. Hanyu, and I. Shiozaki, J. Phys. Soc. Jpn. 64, 934 (1995).

${ }^{13}$ M. Tischer, O. Hjortstam, D. Arvanitis, J. Hunter Dunn, F. May,
K. Baberschke, J. Trygg, J. M. Wills, B. Johansson, and O. Eriksson, Phys. Rev. Lett. 75, 1602 (1995).

${ }^{14}$ H. J. F. Jansen, J. Appl. Phys. 64, 5604 (1988).

${ }^{15}$ C. Liu, E. R. Moog, and S. D. Bader, Phys. Rev. Lett. 60, 2422 (1988).

${ }^{16}$ J. G. Gay and R. Richter, Phys. Rev. Lett. 56, 2728 (1986).

${ }^{17}$ J. G. Gay and R. Richter, J. Appl. Phys. 61, 3362 (1987).

${ }^{18}$ B. Heinrich, K. B. Urquhart, J. R. Dutcher, S. T. Purcell, J. F. Cochran, A. S. Arrott, D. A. Steigerwald, and W. F. Egelhoff, Jr., J. Appl. Phys. 63, 3863 (1988).

${ }^{19}$ G. H. O. Daalderop, P. J. Kelly, and M. F. H. Schuurmans, Phys. Rev. B 42, 7270 (1990).

${ }^{20}$ J. M. MacLaren and R. H. Victora, J. Appl. Phys. 76, 6069 (1994).

${ }^{21}$ B. Úfalussy, L. Szunyogh, P. Bruno, and P. Weinberger, Phys. Rev. Lett. 77, 1805 (1996).

${ }^{22}$ W. Grange, J.-P. Kappler, M. Maret, J. Vogel, A. Fontaine, F. Petroff, G. Krill, A. Rogalev, J. Goulon, M. Finazzi, and N. B. Brookes, J. Appl. Phys. 83, 6617 (1998).

${ }^{23}$ D. M. Eigler and E. K. Schweizer, Nature (London) 344, 524 (1990).

${ }^{24}$ M. J. Zhu, D. M. Bylander, and L. Kleinman, Phys. Rev. B 43, 4007 (1991).

${ }^{25}$ G. Y. Guo, W. M. Temmerman, and H. Ebert, J. Phys.: Condens. Matter 3, 8205 (1991). 
${ }^{26}$ K. Wildberger, V. S. Stepanyuk, P. Lang, R. Zeller, and P. H. Dederichs, Phys. Rev. Lett. 75, 509 (1995).

${ }^{27}$ V. S. Stepanyuk, W. Hergert, P. Rennert, K. Wildberger, R. Zeller, and P. H. Dederichs, Phys. Rev. B 54, 14121 (1996).

${ }^{28}$ S. Blügel, Phys. Rev. Lett. 68, 851 (1992).

${ }^{29}$ H. Beckmann and G. Bergmann, J. Low Temp. Phys. 110, 1173 (1997).

${ }^{30}$ H. Beckmann and G. Bergmann, Phys. Rev. B 54, 368 (1996).

${ }^{31}$ H. Beckmann, R. Schäfer, W. Li, and G. Bergmann, Europhys. Lett. 33, 563 (1996).

${ }^{32}$ S. H. Vosko, L. Wilk, and M. Nusair, Can. J. Phys. 58, 1200 (1980).

${ }^{33}$ B. Drittler, M. Weinert, R. Zeller, and P. H. Dederichs, Phys. Rev. B 39, 930 (1989).
${ }^{34}$ B. Nonas, Ph.D. thesis, Aachen Technical University, 2000.

${ }^{35}$ H. Ebert and M. Battocletti, Solid State Commun. 98, 785 (1996).

${ }^{36}$ M. S. S. Brooks, Physica B 130, 6 (1985).

${ }^{37}$ H. Ebert, R. Zeller, B. Drittler, and P. H. Dederichs, J. Appl. Phys. 67, 4576 (1990).

${ }^{38}$ A. R. Mackintosh and O. K. Andersen, in Electrons at the Fermi Surface, edited by M. Springford (Cambridge University Press, Cambridge, 1980), Chap. The Electronic Structure of Transition Metals.

${ }^{39}$ P. Bruno, Phys. Rev. B 39, 865 (1989).

${ }^{40}$ G. van der Laan, J. Phys.: Condens. Matter 10, 3239 (1998).

${ }^{41}$ B. Nonas, I. Cabria, R. Zeller, P. H. Dederichs, T. Huhne, and H. Ebert, Phys. Rev. Lett. 86, 2146 (2001).

${ }^{42}$ P. Steiner and S. Hüfner, Phys. Rev. B 12, 842 (1975). 